EPJ manuscript No.

(will be inserted by the editor)

\title{
Competition of Brazil nut effect, buoyancy, and inelasticity induced segregation in a granular mixture
}

\author{
Ricardo Brito ${ }^{1}$ and Rodrigo Soto ${ }^{2}$ \\ 1 Departamento de Física Aplicada I and GISC, Facultad de Ciencias Físicas, Universidad Com- \\ plutense, 28040 Madrid, Spain. \\ 2 Departamento de Física, FCFM, Universidad de Chile, Santiago, Chile.
}

\begin{abstract}
It has been recently reported that a granular mixture in which grains differ in their restitution coefficients presents segregation: the more inelastic particles sink to the bottom. When other segregation mechanisms as buoyancy and the Brazil nut effect are present, the inelasticity induced segregation can compete with them. First, a detailed analysis, based on numerical simulations of two dimensional systems, of the competition between buoyancy and the inelasticity induced segregation is presented, finding that there is a transition line in the parameter space that determines which mechanism is dominant. In the case of neutrally buoyant particles having different sizes the inelasticity induced segregation can compete with the Brazil nut effect (BNE). Reverse Brazil nut effect (RBNE) could be obtained at large inelasticities of the intruder. At intermediate values, BNE and RBNE coexist and large inelastic particles are found both near the bottom and at the top of the system.
\end{abstract}

\section{Introduction}

It is known from decades that when a mixture of two types of granular particles are externally excited by means, e.g. a vibration, grains of different size, shape, mass or mechanical properties can mix or segregate. For instance in a mixture of small grains and one large intruder, when vertically vibrated, the intruder can go up [1 or down [2] -the so called direct and reverse Brazil nut effects, respectively. This effect hat has been studied in many papers (see, e.g. Ref. [3] and references therein), where different mechanisms have been proposed to explain the segregation phenomenon. For example, percolation, arching, void filling, convection, interstitial fluid effects, condensation, and some more have been invoked to be the cause of segregation. All these effects are explained in Ref. [3], although the problem is not completely understood yet. When both species have similar sizes (but possibly different) we can select few cases where a variety of segregation mechanisms and scenario appear [4,5,6]. For instance, in Ref. [7] particles of different masses, radii and restitution coefficients are placed in a dish which is horizontally vibrated, finding complete segregation. Segregation is also found in the same geometry when the grains have different friction coefficient with the base [8. Under horizontal swirling, radial segregation of particles of different sizes has been observed 9. In avalanches, grains of different shape segregate in stripes [10; in partially filled rotating drums, axial size segregation develops [11. In two dimensional systems under gravity, sinusoidally vibrated, clustering has been observed [12]. This segregation effect can be modulated by using non-sinusoidal vibration [13.

In some of the cases mentioned above the grain species differ on the friction or restitution coefficient. However few papers have studied segregation when this is the only difference between grains. One of these cases is Ref. [14, where a mixture of spheres that only differ in friction 
coefficients (static, dynamic and rolling) is horizontally vibrated. They find complete mixing -that is, no segregation- for a flat plate while segregation is only observed when the plate was slightly inclined. Therefore, these results contradict the previously mentioned ones.

In a theoretical approach Ref. 15 constructs the hydrodynamic equations from the Boltzmann equation, finding inelasticity induced segregation. The authors explain the phenomenon as a consequence of the temperature gradient in the system induced by inelastic collisions, and relate the concentration gradient with the temperature gradient. In the same spirit, Ref. [16] studies the low density hydrodynamics of a mixture in the so called tracer limit, i.e. where the concentration of one of the components tends to zero. Among other results, they find that the temperature ratio of both species must be a constant. This constant value had been already measured by two experimental groups, both in 3D [17] and 2D [18 and by means of computer simulations in 3D [19] and 2D [20]. They found that the temperature ratio reaches a constant value in the regions of the system where the density is low. No tracer limit is needed. Generalization to moderate density has been done by Garzó [21] based on a kinetic approach using the Enskog equation for dissipative hard spheres.

Recently, it has been shown numerically that in moderately dense vibrofluidized granular matter, inelasticity induced segregation takes place [22]. When a mixture of grains of equal size and mass, but differing in their restitution coefficient in put in a vibrating box, the more inelastic grains sink, segregating partially with the other species. In this paper we study if this inelasticity induced segregation can compete with two other known mechanisms of grain segregation: buoyant forces when grain densities differ and the Brazil nut effect when their sizes differ.

The structure of this paper is as follows. In Section 2 we describe the system under consideration. Section 3 reproduces the results presented in Ref. 22 regarding the macroscopic segregation of two species differing only in their restitution coefficient. Section 4 analyzes the competition with buoyant forces, when considering grains of equal size, but different masses and restitution coefficients. Section 5 studies the competition with the Brazil nut effect when grains of the two species have the same density, but differ in size and restitution coefficients. We conclude with Section [6] summarizing the results of the paper.

\section{Description of the system}

We study the effect of the difference on restitution coefficients in the segregation phenomenon, by means of Molecular Dynamics simulations of a bidimensional granular mixture of two types of particles, $A$ and $B$. Grains are modeled as smooth Inelastic Hard Disks, but differing on the normal restitution coefficient that characterizes their inelastic collisions. The two species can also differ in their masses $m_{A}$ and $m_{B}$, and in their diameters $\sigma_{A}$ and $\sigma_{B}$. The restitution coefficient for $A$ - $A$ collisions is $\alpha_{A}$, for $B-B$ collisions is $\alpha_{B}$. For the interparticle collisions $A-B$ we have taken $\alpha_{A B}=\left(\alpha_{A}+\alpha_{B}\right) / 2$. Usually we will consider that $\mathrm{B}$ are the most inelastic particles $\left(\alpha_{B}<\alpha_{A}\right)$.

We have taken a fixed total number of particles $N_{T}=N_{A}+N_{B}$, changing the concentration of the $B$ particles. For the simulations reported in this paper, we have fixed $N_{T}=680$ disks and varied $N_{B}$ from 10 (that can be considered as a tracer limit) until 160. The disks are placed under the action of a gravitational acceleration $g$ pointing downward in a rectangular box of width $L_{x}=50 \sigma_{A}$, infinite height, and with the bottom wall oscillating periodically at high frequency $\omega$ and small amplitude $A$, with a bi-parabolic waveform [23. Periodic boundary conditions are used in the horizontal direction. They avoid the appearance of convective rolls by the influence of the walls. Under these conditions, the system reaches a stationary state with gradients in the vertical direction [24].

Units are chosen such that $\sigma_{A} \equiv \sigma=1, m_{A}=1$ and $k_{B}=1$. The time scale is fixed by the characteristic energy of wall oscillation, that is $m_{A}(A \omega)^{2}=1$. Simulations are performed with a fixed value of the gravity acceleration $g$ in order to reduce the parameter space and provide a more detailed analysis on the effect of inelasticity. The value of $g=0.15$ (in our units), was chosen because the associated value of the ratio of the acceleration induced by the vibration 
versus the gravity, $\Gamma=\left(A w^{2}\right) / g$ can be easily reached experimentally, as it takes the value of $\Gamma \simeq 6.67$. Finally, the amplitude on the vibration is set to $A=0.01 \sigma$.

\section{Inelasticity induced segregation}

In this section we reproduce the main results concerning the macroscopic segregation of the two species when they only differ in restitution coefficient, that were already reported in Ref. 22. First, to illustrate the main observed features, we report results of a simulation having a small fraction of inelastic particles $N_{B}=10$, and $\alpha_{B}=0.7$ and the rest nearly elastic: $N_{A}=670$, $\alpha_{A}=0.98$.

The density profiles of the two species are shown in Fig. 1a, where we plot the number density of particles of type $A$ and $B: n_{A}(z)$ and $n_{B}(z)$. The normalization of these quantities is such that $\int_{0}^{\infty} d z \int_{0}^{L x} d x n_{A}(z)=N_{A}$ (resp. $B$ ). For plotting purposes only, $n_{B}$ is rescaled by a factor $N_{A} / N_{B}$, so in the case of no segregation both profiles would be identical. Both densities have the characteristic shape of vibrofluidized systems subject to gravity: there is a initial density increase due to the abrupt temperature drop caused by dissipation, and at higher positions, density decreases again due to gravity [24]. Density exhibits a maximum at $z \simeq 15 \sigma$ where the density $n \simeq 0.5$, so the system cannot be considered as dilute. The density profile of the more inelastic particles, $B$, is plotted as a dotted line in Fig 1a. Its maximum is located at smaller $z$, indicating that they are closer to the bottom of the container as compared to the more elastic ones. The segregation is not complete, though.

The temperature profiles are also highly inhomogeneous, as shown in Fig. 2 a. For both species, the temperature presents a initial abrupt drop, but later (after $z \simeq 20 \sigma$ ) both profiles present a linear increase with height. This phenomenon was already observed in one-component systems, and it is associated to the energy transport term, $-\mu \nabla n$, related to density gradients, that appear in granular fluids 25. Let us note that the maximum density does not coincide with the temperature minimum: there is a shift between these two quantities which is qualitatively described by a hydrostatic balance in presence of gravity [25, 15].
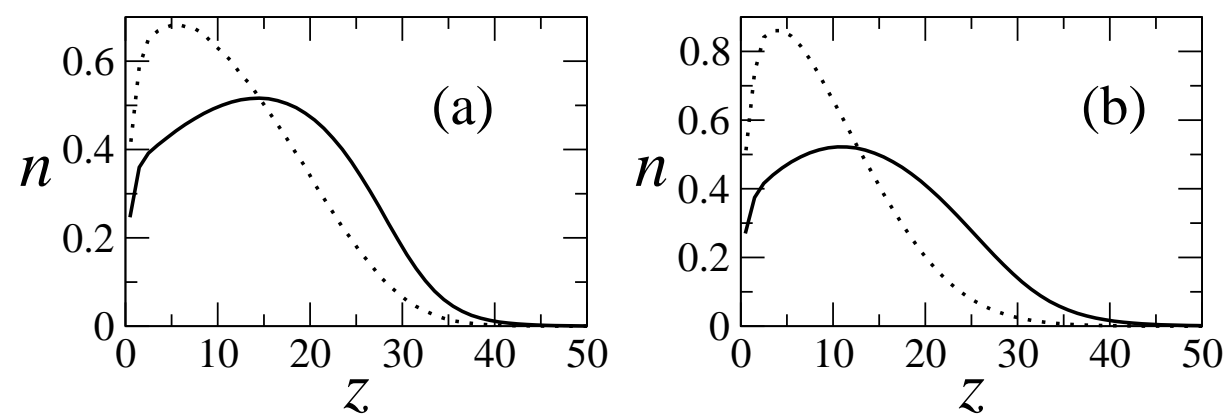

Fig. 1. Density profiles for the two species, density of $A, n_{A}$, (solid line) and the rescaled density of $B, n_{B} N_{A} / N_{B}$, (dotted line). (a): both species are inelastic with $N_{A}=670, N_{B}=10, \alpha_{A}=0.98$, $\alpha_{B}=0.7$. (b): $A$ is elastic while $B$ is inelastic and $N_{A}=640, N_{B}=40$, and $\alpha_{B}=0.5$

The described segregation of species $A$ and $B$ is produced by their different restitution coefficients as all the other properties are the same. To study in more detail the effect of the difference of inelasticities, we proceed to study the limiting case in which the $A$ particles are elastic $\left(\alpha_{A}=1\right)$ and only the $B$ particles are inelastic (and consequently, collisions $A-B$ are also inelastic). In this way we also limit the parameter space, allowing to a more detailed quantitative study.

Figures 1b and 2b show the density and temperature profiles for such case, where particles of type $A$ are elastic $\left(\alpha_{A}=1\right)$ particles $B$ are inelastic $\left(\alpha_{B}=0.5\right)$ considering $N_{B}=40$. It is observed that the main properties of the profiles are preserved, even the positive slope of 

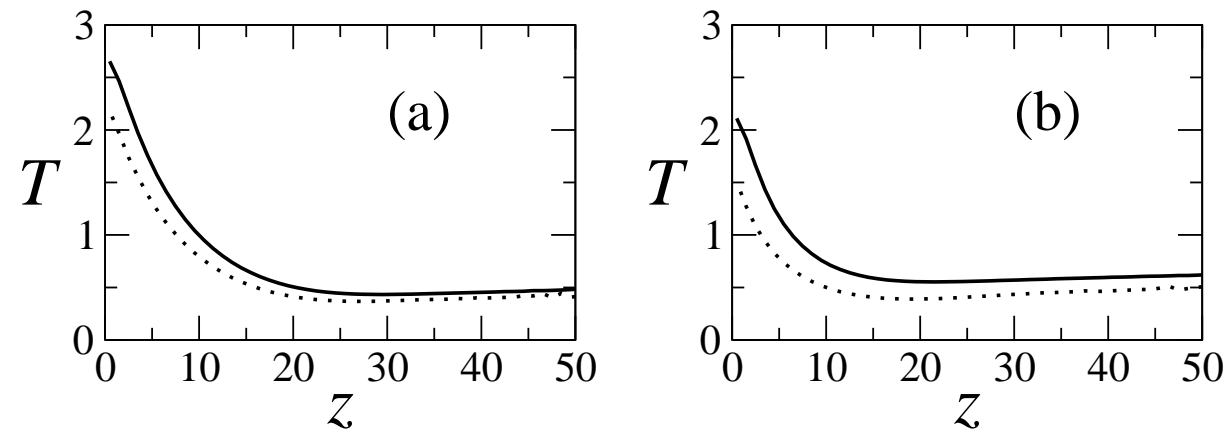

Fig. 2. Temperature profiles for two species, $T_{A}$ (solid line) and $T_{B}$ (dotted line). (a) and (b) graphics have the same parameters as in Fig. 1

$T_{A}$ despite the A-A collisions are elastic. Partial segregation is again observed, where inelastic particles, $B$, sink to the bottom of the container while elastic ones, $A$, are majority at upper layers of the fluid.

These results are not surprising in view of the predictions of Ref. [15, where it is argued that the segregation is produced when the particles with different restitution coefficients are immersed in a temperature gradient. The gradient is induced by the inelastic collisions, so such gradient can be created vibrating a mixture of elastic and inelastic particles. The latter ones dissipate some of the energy injected by vibration creating a stationary state. The hydrodynamic description of the mixture also contains the dissipative flux $-\mu \nabla n$, and therefore it is expected that the hydrodynamic profiles of density and temperature will be equivalent to a full inelastic system.

Figure 3 shows the temperature ratios $T_{B}(z) / T_{A}(z)$ for the simulations described in Fig. 2. At low densities it was found experimentally [17,18] and by employing kinetic theory [16] that such temperature ratio must be constant. However, we find a non constant ratio in the $z$ direction that only agrees with the result of 16 at high $z$, say for $z$ larger than 30 . Inspection of Fig. 1 shows that the density at $z>30$ is smaller than 0.2 , so we can consider that the system is in a low density regime. Therefore the approximations of 16 are valid. In the case where A particles are elastic, equivalent predictions for the temperature ratio were given in [26, 27].

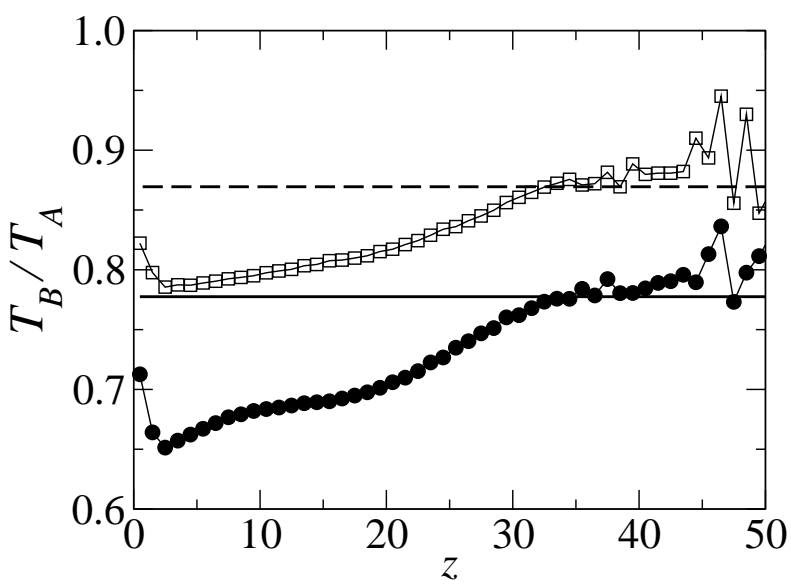

Fig. 3. Temperature ratios $T_{B}(z) / T_{A}(z)$ for the simulations described in Fig. 2. Simulation results (solid circles) and theoretical prediction in [16] (solid line) for $N_{A}=670, N_{B}=10, \alpha_{A}=0.98$, $\alpha_{B}=0.7$. Simulation results (open squares) and theoretical prediction in 16] (dashed line) for A elastic, $N_{A}=640, N_{B}=40$, and $\alpha_{B}=0.5$ 
In order to quantify the segregation, a series of simulations are performed with $N_{B}$ ranging from 10 to 160, and $\alpha_{B}$ between 0.2 and 0.9. Larger values of $N_{B}$ or smaller restitution coefficients lead to clustering as described in [28. For each simulation we compute the segregation parameter, defined as:

$$
\delta=1-\int d z n_{A}(z) n_{B}(z) / \sqrt{\int d z n_{A}^{2}(z) \int d z n_{B}^{2}(z)}
$$

where the $n_{A}(z)$ and $n_{B}(z)$ are the local density, as plotted in Fig. 1. The segregation parameter is bounded between 0 and 1 . The value $\delta=1$ corresponds to complete segregation, as $\delta$ is 1 if $n_{A}(z)$ and $n_{B}(z)$ do not overlap. On the contrary, $\delta=0$ means complete mixing, as this value can only be obtained if $n_{B}(z)$ is proportional to $n_{A}(z)$.

The results for $\delta$ are collected in Fig. 4 where the quantity $\delta$ is plotted versus the coefficient $\alpha_{B}$ for different values of $N_{B}$. The fact that $\delta$ is always non vanishing confirms that the segregation exists whenever the restitution coefficients are different. Only in the case when $\alpha_{B} \rightarrow 1$ the quantity $\delta$ approaches 0 , limit in which there is no segregation. Note that, for each $\alpha_{B}, \delta$ increases with $N_{B}$. The results confirm that segregation is not complete as $\delta$ never gets close to 1 . In addition, for each simulation, the center of mass of the $A$ and $B$ species are computed, $Z_{A / B}$, finding that $Z_{A}>Z_{B}$ in all cases.

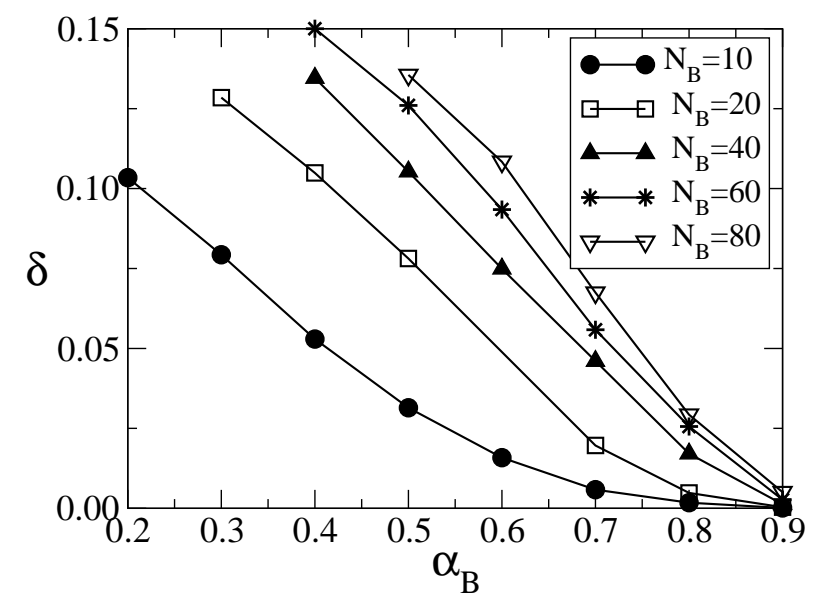

Fig. 4. Segregation parameter $\delta$ as a function of $\alpha_{B}$ for $\alpha_{A}=1.0$ and $N_{T}=640$. Different curves correspond to various concentrations of $B$ particles. In the elastic limit $\alpha \rightarrow 1$ all curves coincide at $\delta=0$.

The reported inelasticity induced segregation have a microscopic origin as stated in Ref. 22]: the most inelastic particles induce locally a region of high density and low temperature, resembling a cold droplet that falls in a gravitational field. The droplet is created by the dissipation in a way that resembles the clustering instability of the granular gases. Quantitative proof of this argument can be found in Ref. [22], by studying the density and temperature pair distribution functions of the $A$ and $B$ particles.

\section{Competition with buoyancy forces}

Up to now, we have considered a mixture where grains of both species have the same masses and diameters. The main conclusion so far is that the inelasticity can create an effective force, pointing downwards, that sinks the more inelastic particles to the bottom of the container. Besides, when densities differ, either because the mass and/or the diameters of the particles are different, buoyancy forces appear making lighter grains move to the top. It can be asked whether 
the inelasticity induced segregation could compensate the buoyancy force experienced by lighter $B$ particles. To confirm or deny this idea, a series of simulations with equal sized particles $\left(\sigma_{B} / \sigma_{A}=1\right)$, keeping fixed $\alpha_{A}, N_{A}$, and $N_{B}$, but varying $m_{B} / m_{A}$ and $\alpha_{B}$ is performed. In order to determine which force dominates, we compute the center of masses of the $A$ and $B$ particles, denoting them by $Z_{A}, Z_{B}$. Their precise definition is:

$$
Z_{A}=\frac{\int_{0}^{\infty} d z z n_{A}(z)}{\int_{0}^{\infty} d z n_{A}(z)}=\frac{\int_{0}^{\infty} d z z n_{A}(z)}{N_{A} / L_{x}}, \quad(\operatorname{resp} . B)
$$

If $Z_{A}>Z_{B}$ particles of type $A$ will be, on average, closer to the top of the container than particles of type $B$ and viceversa. The ratio of $Z_{B} / Z_{A}$ being larger or smaller than one will be our criterion for deciding which force actually dominates.

The results for $\alpha_{A}=1, N_{A}=640$, and $N_{B}=40$ are presented in Fig. 5. The mass ratio $m_{B} / m_{A}$ is varied from 0.3 until 1 , and the inelasticity of the $B$ particle from 0.4 until 0.9. For each value of $\alpha_{B}$, there is a particular value of the mass ratio, $\left(m_{B} / m_{A}\right)^{*}$ at which the positions of the two center of masses coincide. The inelastic particles have lower center of mass if $m_{B} / m_{A}>\left(m_{B} / m_{A}\right)^{*}$, and therefore sinking due to inelasticity wins to the buoyancy force in this range. On the contrary, buoyancy force dominates if $m_{B} / m_{A}<\left(m_{B} / m_{A}\right)^{*}$ and lighter and inelastic particles go up.

Figure 5 also presents the value of the critical ratio $\left(m_{B} / m_{A}\right)^{*}$ as a function of $\alpha_{B}$ : more inelastic particles have a higher tendency to sink and therefore a stronger mass ratio is needed to compensate the inelasticity induced segregation. Note that when $\alpha_{B} \rightarrow 1$, the positions of both center of masses diverge because a vibrated elastic medium does not have a stationary state. There is an indication of such divergence in the steep slope of the dashed line in the upper left panel of Fig. 5. The segregation parameter $\delta$ does not vanish for any value of $m_{B} / m_{A}$, indicating that there is no complete mixing even at the value of $m_{B} / m_{A}=\left(m_{B} / m_{A}\right)^{*}$, where both center of masses coincide. The value of $\delta$ (not shown here), however, is minimum at this precise mass ratio. Also, $\delta$ does not reach 1 either, so no complete segregation is observed.

A second series of simulations were done with $\alpha_{A}=0.9$ and are presented in Fig. [5] The qualitative features are similar to the the previous case $\left(\alpha_{A}=1.0\right)$, except for some remarks. The first one in the center of mass of species $A$ is about constant, independent of the mass ratio or the dissipation parameter of particles $B$. This is so because the particles $A$ are inelastic and dissipate a great deal of energy, forming a very dense system. This density is near the close packing, as it will be illustrated in the forthcoming figures. The second remark is that, as opposite to the $\alpha_{A}=1$ case, the center of masses do not diverge when $\alpha_{B} \rightarrow 1$ due to the finite inelasticity of the $A$ particles. Finally, as $\alpha_{A}=0.9$, the ratio $\alpha_{B} / \alpha_{A}$ can exceed one. In particular, we can make the $B$ particles elastic by choosing $\alpha_{B}=1 / 0.9=1.1111 \ldots$. We have done so and included the results in the bottom part of Fig. 5 . As expected, particles $B$ must be heavier than $A$ in order to compensate the effective upwards force produced because they are more elastic. In this particular case the value of $\left(m_{B} / m_{A}\right)^{*} \simeq 1.17$.

\section{Competition with the Brazil nut effect}

When grains are mixed such that they differ in size, a remarkable phenomenon takes place: the larger grains rise to the top even if they are denser. This phenomenon, called the Brazil nut effect (BNE), has been widely studied in order to identify the different mechanisms that produce it and also to characterize the range of parameters where it takes place. As mentioned in the introduction, it has been found that besides the normal BNE, it is also possible that, under certain conditions, the large particles sink in the so-called reverse Brazil nut effect (RBNE). In its simpler form, the BNE is studied in the neutrally buoyant case, where the mass ratio between the species equals their volume ratio.

The results of the previous sections suggest that if the larger particle is also more inelastic the inelasticity induced segregation can compete with the BNE. We have performed a series of simulations of a neutrally buoyant mixture (i.e., $\left.m_{B} / m_{A}=\left(\sigma_{B} / \sigma_{A}\right)^{2}\right)$ where we have varied the 

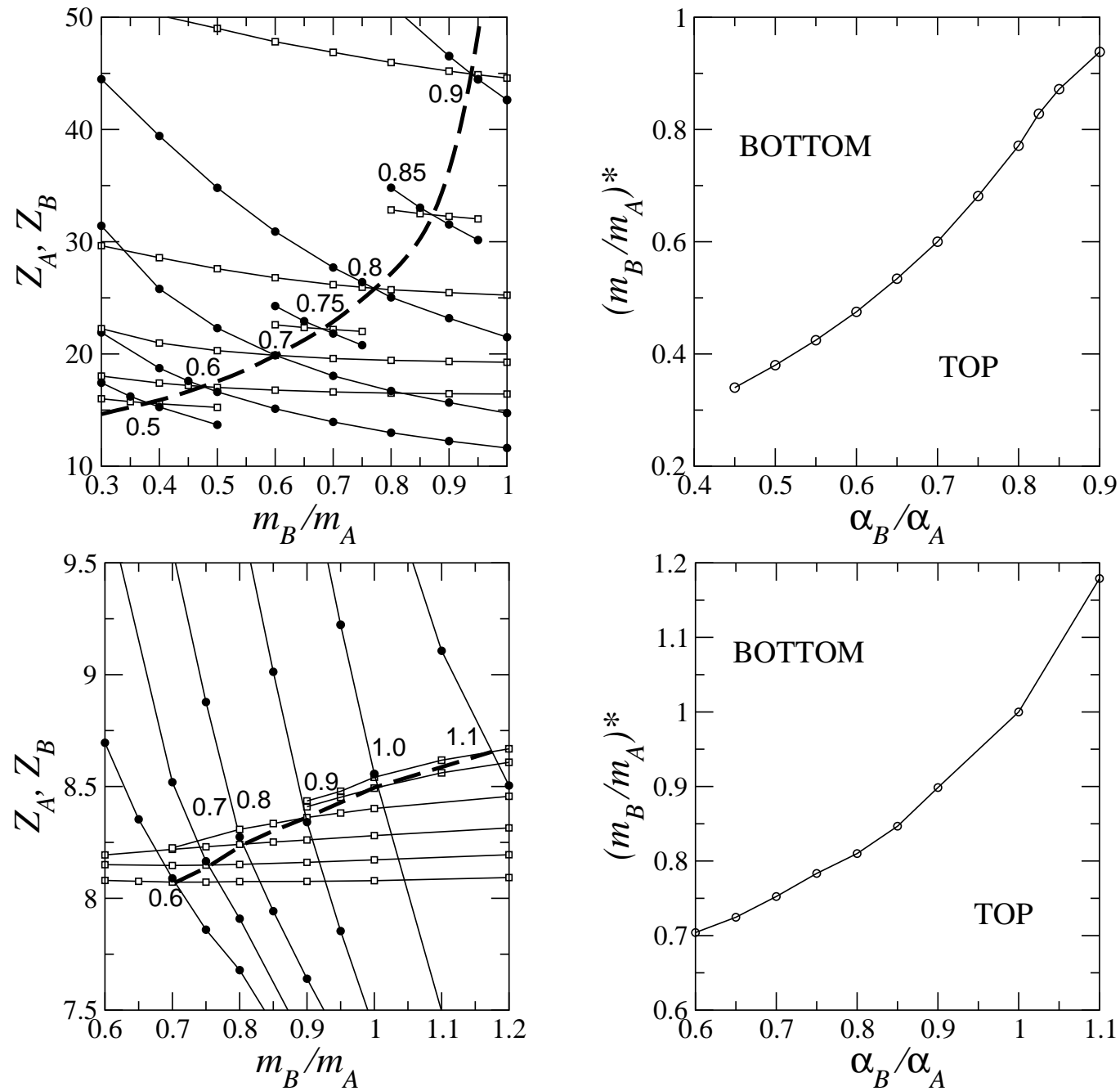

Fig. 5. Left: Position of the center of mass of $A$ (open squares) and $B$ particles (solid circles) as a function of the relative mass of $B$ particles $m_{B} / m_{A}$ for different values of the ratio of the restitution coefficients, $\alpha_{B} / \alpha_{A}$ (indicated in the plot). The locus of the mass ratio at which the two centers of mass coincide is indicated by a thick dashed line. Right: Critical mass ratio $\left(m_{B} / m_{A}\right)^{*}$ at which the two centers of mass coincide as a function of the ratio of the restitution coefficients $\alpha_{B} / \alpha_{A}$. The regions in the parameter space where $B$ particles move to the top or to the bottom are indicated by the respective labels in the plot. Simulation parameters are fixed to $N_{A}=640, N_{B}=40, \sigma_{B} / \sigma_{A}=1$, and $\alpha_{A}=1$ (top) and $\alpha_{A}=0.9$ (bottom).

size of $B$ particles $\sigma_{B}$ and the restitution coefficients $\alpha_{A}$ and $\alpha_{B}$, while the number of particles are fixed to $N_{A}=640$ and $N_{B}=40$. This parameter space is huge as we have restricted to B particles that are not too large; specifically we have considered $1 \leq \sigma_{B} \leq 2.5$.

First, we consider the usual BNE case with $\alpha_{A}=\alpha_{B}$ and $\sigma_{B}=1.5,2.0$ (that is, the two species only differ in their sizes). At low dissipation $\left(\alpha_{A}=\alpha_{B}=0.95\right)$ there is no BNE but rather the two species mix with $B$ particles having a narrower distribution near the center (see Fig. 6). When the dissipation is higher $\left(\alpha_{A}=\alpha_{B}=0.9,0.85\right)$ the larger particles sit on the top as in the usual BNE (see Fig. 6). The segregation is partial when $\sigma_{B}=1.5$ but it is complete when $\sigma_{B}=2.0$, differently as in the other cases, where segregation was always partial. In summary, we have checked that the BNE can be obtained with moderate size difference as long as the inelasticity is strong enough. 
When the two inelasticities differ (with $B$ particles being larger in size and more inelastic), it is expected that the BNE could be compensated by the inelasticity induced segregation. A series of simulations with $\alpha_{A}=1, \sigma_{B}=2.0$, and different values of $\alpha_{B}$ show that the inelasticity induced segregation is the dominant mechanism and $B$ particles sink (that is, RBNE is found) for large inelasticities and when the elastic limit is approached both species mix (see Fig. 7). However, the elastic limit could not be achieved because, when both species are elastic, the vibrated granular gas has no stationary state.

A different situation is found when $\alpha_{A}=0.9$ and $\sigma_{B}=1.5$, where a transition from RBNE to BNE is obtained with coexistence of both states. The density profiles for different values of $\alpha_{B}$ are presented in Fig. 8. When both inelasticities are equal or the $B$ particles are slightly more inelastic than $A$ particles, a clear BNE is found. But when the $B$ inelasticity is increased, an important fraction of $B$ particles sink showing a coexistence of BNE and RBNE. In fact, a "sandwich" configuration is obtained with $B$ particles being in the bottom and top layers while $A$ particles are in the middle region. The segregation, as is common in all the examples presented here, is not complete and particles have a partial mixing. An stronger separation has been found experimentally [29] in a granular mixture of glass and bronze particles vertically shaked. These authors found, however, complete segregation.

If we define the transition from BNE and RBNE as the dissipation when $Z_{A}=Z_{B}$, we find a value for $\alpha_{B} \simeq 0.71$. In other words, when $\sigma_{B}=1.5$, a dissipation $\alpha_{B}<0.71$ makes the $B$ particles to sink. As mentioned before, the parameter $\delta$, defined in Eq. (1), takes its minimum value: $\delta=0.053$.

The coexistence of BNE and RBNE could be a transient configuration due, in part, to the high compacity of the small grains in the middle region that limits the mobility of the large particles. They could not migrate between the bottom layers and the top ones. A dynamic
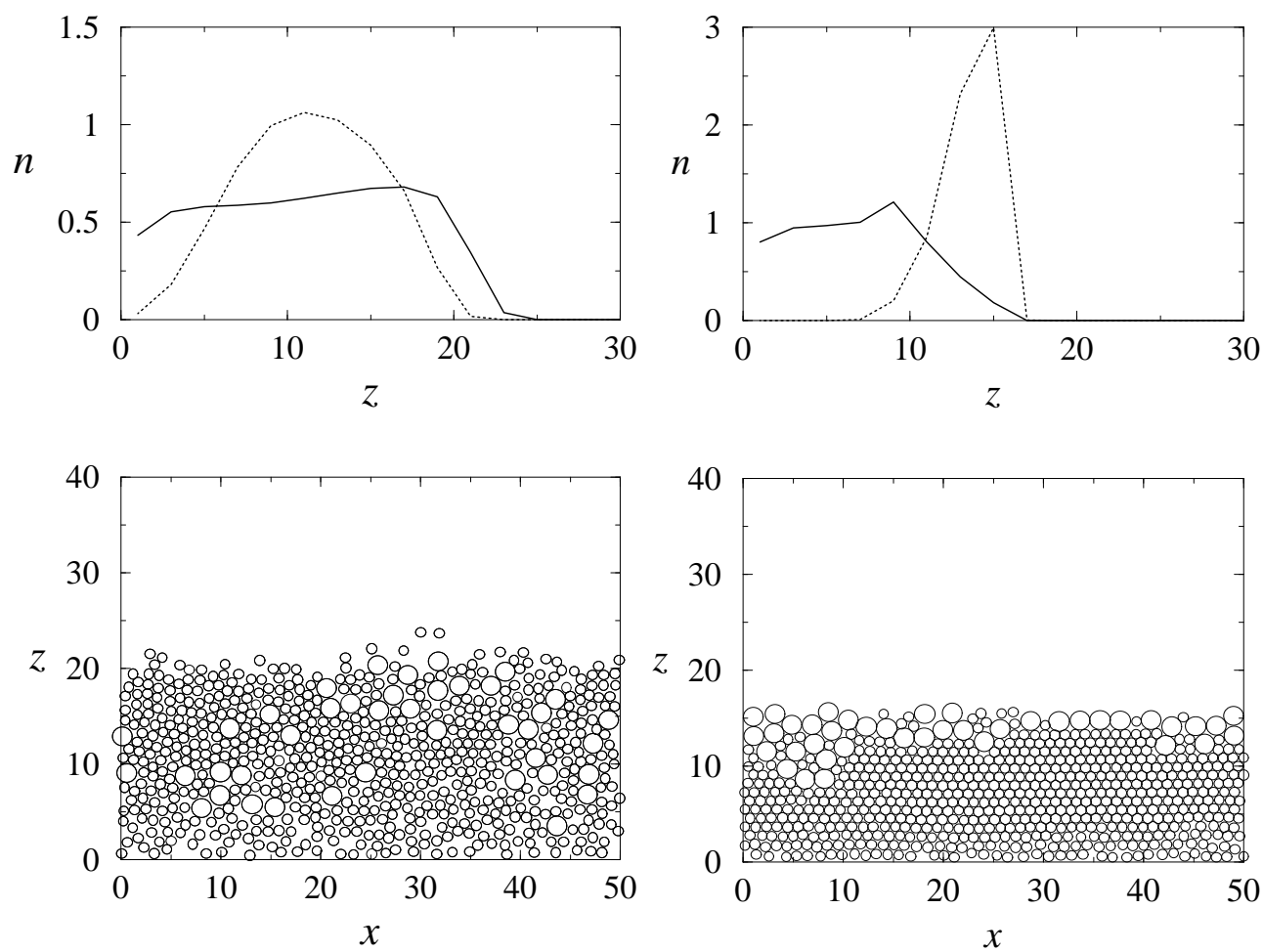

Fig. 6. Left: Top: Density profiles of $A$ particles (solid line) and $B$ particles (dashed line) in a neutrally buoyant mixture with $\sigma_{B}=2.0, N_{A}=640, N_{B}=40$, and $\alpha_{A}=\alpha_{B}=0.95$ (left) and $\alpha_{A}=\alpha_{B}=0.85$ (right). Bottom: Configuration of the grains in the two systems. 

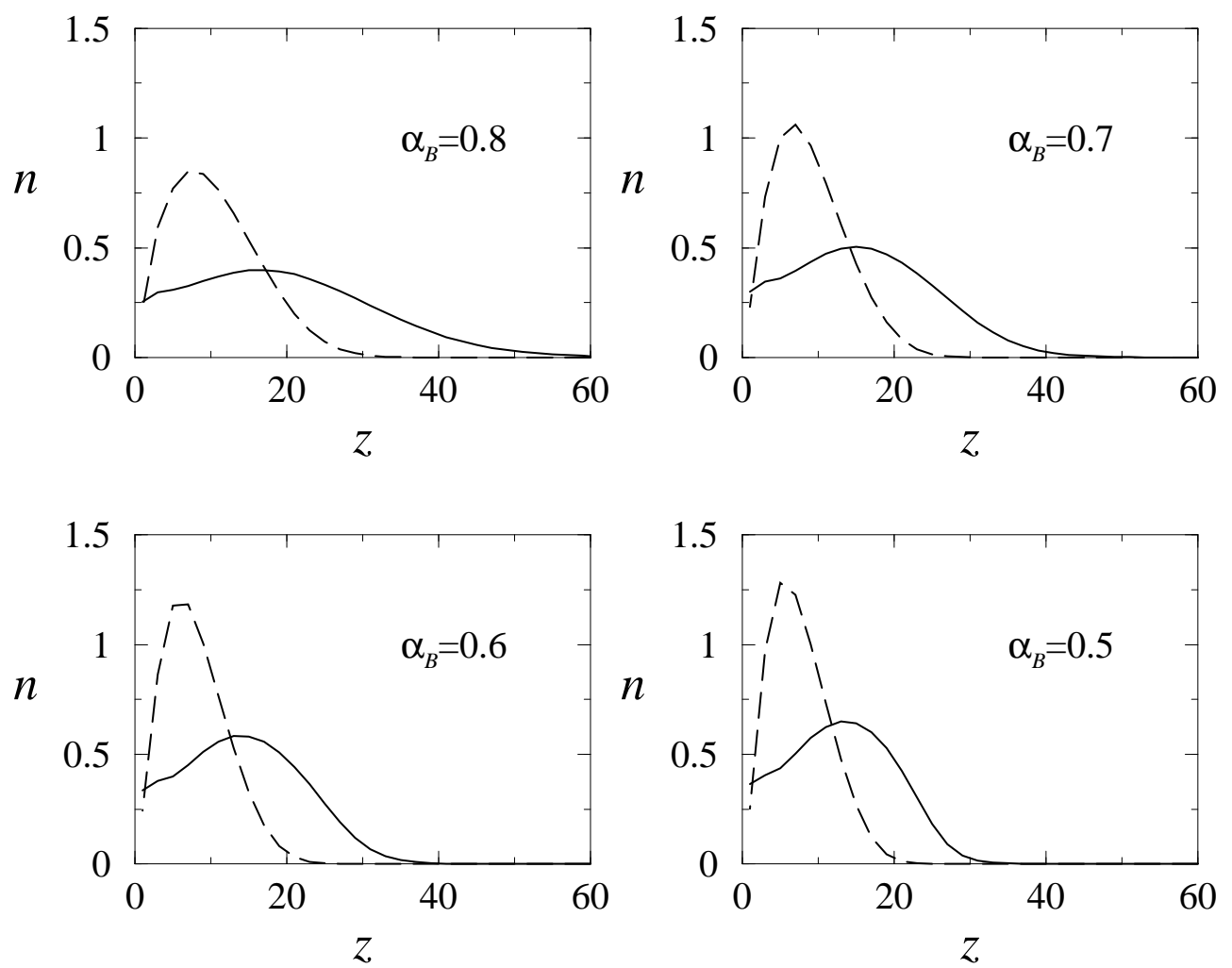

Fig. 7. Density profiles of $A$ particles (solid line) and $B$ particles (dashed line) in a neutrally buoyant mixture with $\alpha_{A}=1, \sigma_{B}=2.0, N_{A}=640, N_{B}=40$, and different values of $\alpha_{B}$ (indicated in the figures).

analysis, that is beyond the purpose of this article, could lead some insight into the coexistence of both effects.

An inspection of the particle configurations with $\alpha_{B}=0.3$ where both RBNE and BNE coexist, shows that $B$ particles develop a tendency to form pairs and small clusters (see Fig.9). This phenomenon was already reported in the case of particles of equal size and it was quantitatively characterized in terms of the pair distribution functions [22]. There is not only a macroscopic segregation described in terms of the density profiles and center of mass positions, but also a microscopic segregation with a tendency of the more inelastic particles to aggregate in small clusters. In this case, the pair distribution function of the $A A$ particles resembles the structure of a hexagonal crystaline structure, while the $B B$ pair distribution function has a very high peak at $\sigma_{B}$, followed by a much smaller peak and hardly any structure for distances larger than $2 \sigma_{B}$.

We have shown that inelasticity induced segregation can compete with both buoyant forces and the BNE, separately. If a mixture of grains is considered such that $B$ particles have the same mass as $A$ particles, but have larger size, both buoyancy forces and the BNE push $B$ particles to the top. The simulation results presented in Fig. 10 show that for $\sigma_{B} \gtrsim 1.9$ buoyancy and BNE cannot be compensated by inelasticity and the large grains go always to the top. When $\sigma_{B} \lesssim 1.9$ inelasticity induced segregation can balance the the combined effect of buoyancy and $\mathrm{BNE}$ and higher inelasticities are needed to compensate their effects for larger $B$ particles.

The temperature profiles present the same qualitative behavior as in the case of a mixture where grains differ only in their restitution coefficient (see Fig. 111). That is, an initial abrupt drop, followed by a slight linear increase with height. The temperature ratio $T_{B}(z) / T_{A}(z)$ shows that $B$ particles are always colder than $A$ particles but it does not agree with kinetic theory 

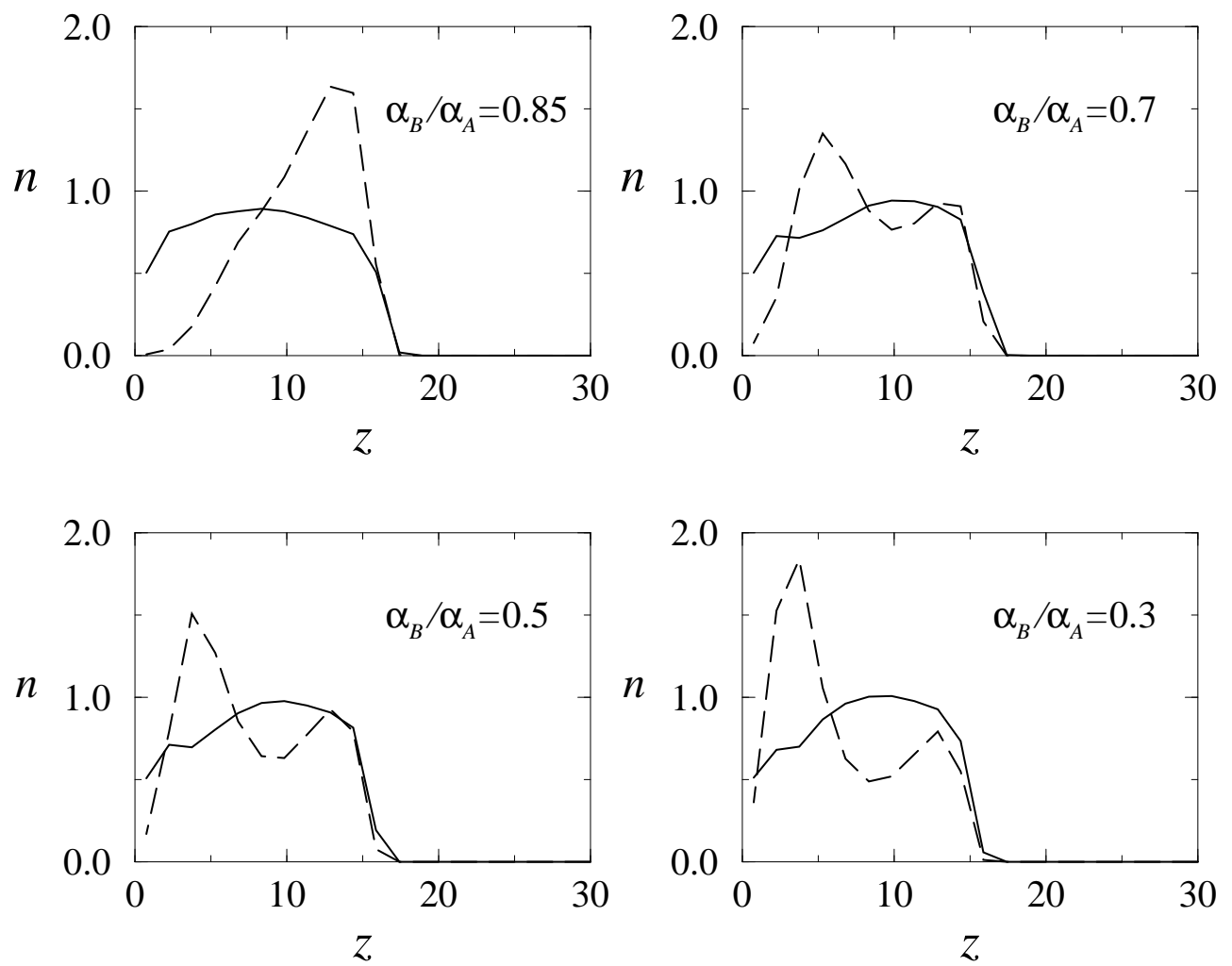

Fig. 8. Density profiles of $A$ particles (solid line) and $B$ particles (dashed line) in a neutrally buoyant mixture with $\alpha_{A}=0.9, \sigma_{B}=1.5, N_{A}=640, N_{B}=40$, and different values of $\alpha_{B}$ (indicated in the figures).

predictions for dilute and moderately dense granular gases [16] as we work in a high density regime, close to close packing in some of our simulations.

\section{Conclusions}

The main conclusion of the present paper is that different restitution coefficients alone create segregation in a binary mixture vertically vibrated. This segregation mechanism can compete or enhance other segregation mechanisms as buoyancy or the Brazil nut effect. The restitution coefficients must be considered, in addition to the usual material properties (mass ratio and diameter ratio), in order to describe accurately the segregation process in a granular mixture. The effect of the inelasticity is such that the most inelastic particles sink to the bottom of the container while the less inelastic ones raise to the top. Segregation is not complete, however, but only partial. The density profiles of each species show a maximum which are located in different positions depending on the inelasticities, but they have an important overlap.

The segregation effects presented here also appears by vibrating a mixture of elastic and inelastic particles. Again inelastic particles migrate to the bottom of the container and elastic one prefer the upper part. This phenomenon allows to make a systematic study of the mechanism reducing the parameter space.

If, besides the difference of inelasticities, the two species differ in mass density buoyancy forces enter into play. If the inelastic particles are less dense, the two mechanism compete and a transition line in the parameter space is found that distinguish the cases where the inelastic particles have lower or higher center center of mass compared to the elastic ones. 


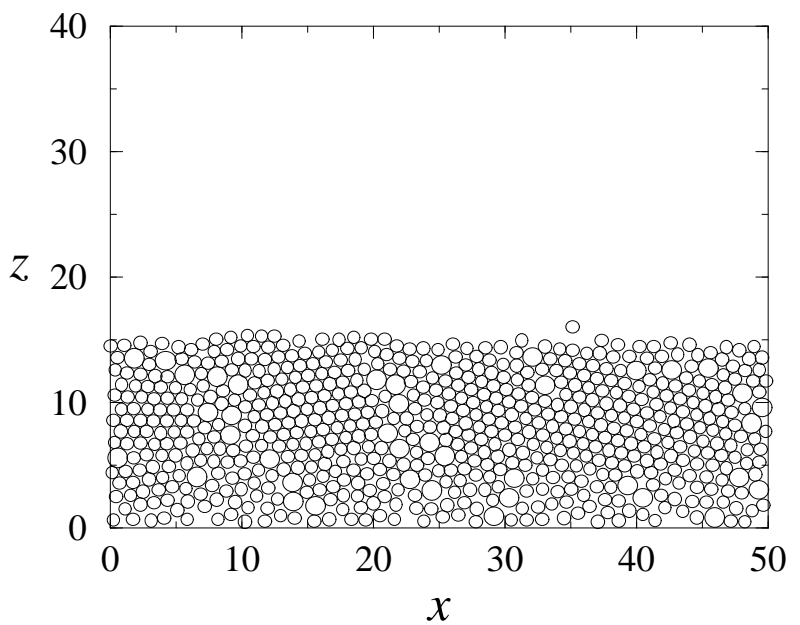

Fig. 9. Configuration of the grains with $\alpha_{A}=0.9, \alpha_{B}=0.3, \sigma_{B}=1.5, N_{A}=640$, and $N_{B}=40$. The large and more inelastic grains aggregate in small clusters.
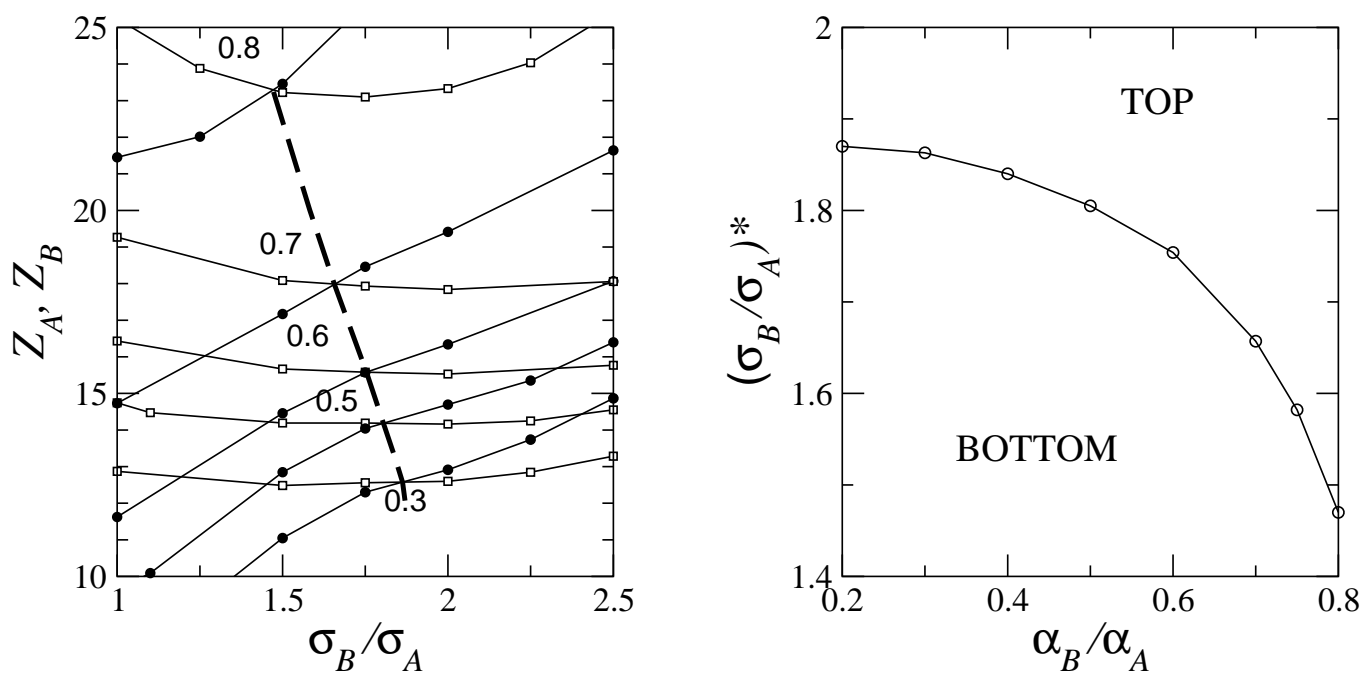

Fig. 10. Left: Position of the center of mass of $A$ (open squares) and $B$ particles (solid circles) as a function of the relative size of $B$ particles $\sigma_{B} / \sigma_{A}$ for different values of $\alpha_{B}$ (indicated in the plot). The locus of the mass ratio at which the two centers of mass coincide is indicated by a thick dashed line. Right: Critical size ratio $\left(\sigma_{B} / \sigma_{A}\right)^{*}$ at which the two centers of mass coincide as a function of the inelasticity of $B$ particles, $\alpha_{B} / \alpha_{A}$. The regions in the parameter space where $B$ particles move to the top or to the bottom are indicated by the respective labels in the plot. Simulation parameters are fixed to $\alpha_{A}=1.0, N_{A}=640, N_{B}=40$, and $m_{B}=1$.

More inelastic particles need higher buoyancy forces to compensate the inelasticity induced segregation mechanism.

When the two species have the same density (that is, they are neutrally buoyant) but differ in their size, the Brazil nut effect appears: large particles have the tendency to go to the top. When the large particles are also more inelastic, the two mechanism compete. The simulations show that there is a range of parameters where a large number of the large and inelastic grains are simultaneously at the top and at the bottom: Brazil nut effect and the reverse Brazil nut effect coexist. The relative proportion of the particles in the two regions depend on the control parameter. 

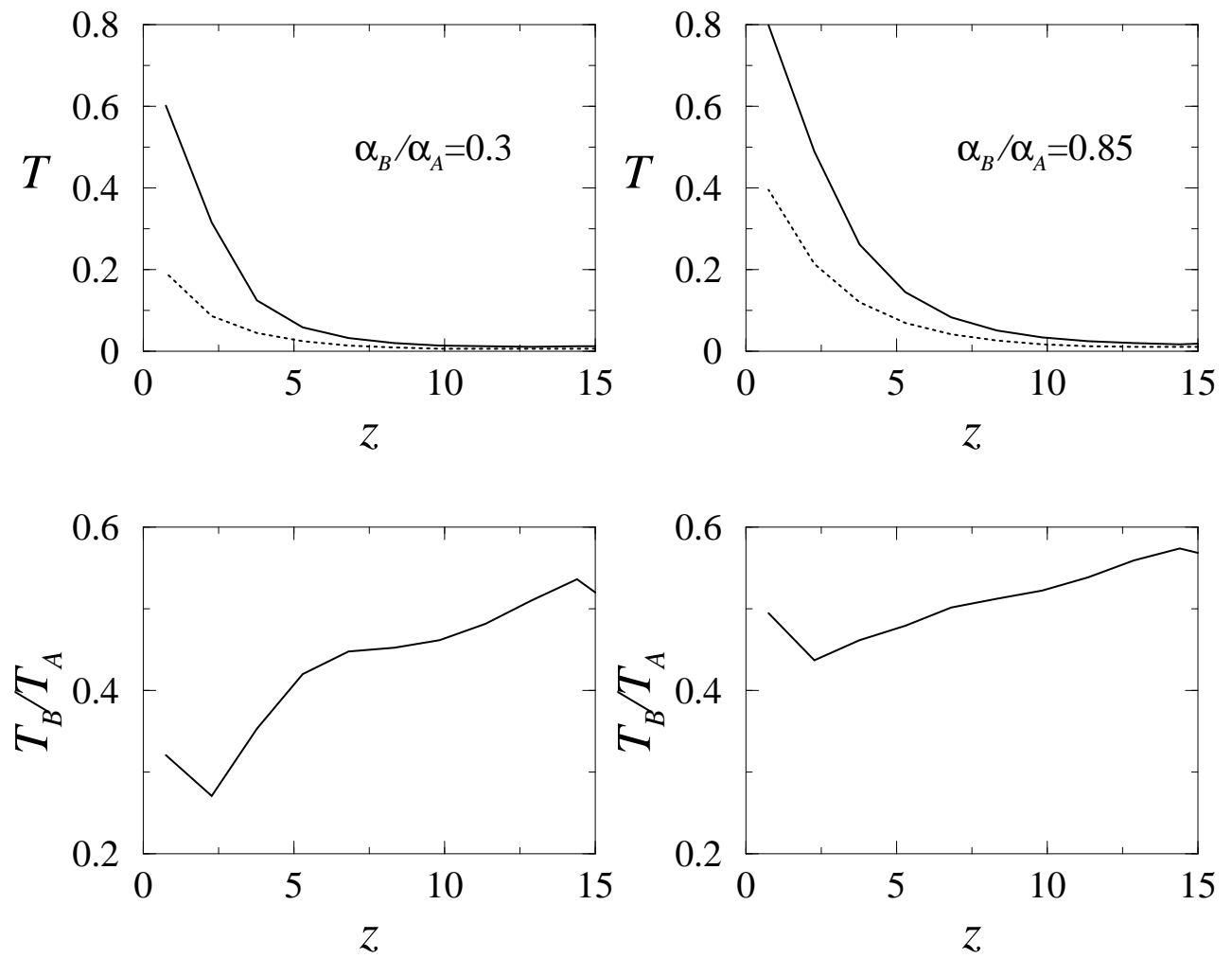

Fig. 11. Temperature profiles (top) of $A$ particles (solid line) and $B$ particles (dotted line) and temperature ratios $T_{B}(z) / T_{A}(z)$ (bottom) for $\alpha_{B}=0.3$ (left) and $\alpha_{B}=0.85$ (right). Simulations were done with $\alpha_{A}=0.9, \sigma_{B}=1.5, N_{A}=640, N_{B}=40$, and $m_{B} / m_{A}=2.25$ (neutrally buoyant).

Concerning the temperatures, most dissipative particles have a lower temperature than the most elastic ones, both having the characteristic shape of vibrofluidized system (fast cooling away from the moving boundary followed by a heating that grows linearly with the distance). Their rate, however, does not agree with kinetic theory predictions for dilute and moderately dense granular gases.

We want to thank J.M.R. Parrondo for very useful comments. R.B. is supported by the Spanish Projects MOSAICO, UCM/PR34/07-15859 and the Program Profesores UCM en el Extranjero. The research is supported by Fondecyt grants 1061112, 1070958, and 7070301 and Fondap grant 11980002.

\section{References}

1. A. Rosato, K.J. Strandburg, F. Prinz, and R.H. Swendsen, Phys. Rev. Lett. 58, 1038 (1987).

2. A. P. J. Breu, H.-M. Ensner, C. A. Kruelle, and I. Rehberg, Phys. Rev. Lett. 90, 014302 (2003).

3. A. Kudrolli, Rep. Prog. Phys. 67, 209 (2004).

4. D. A. Huerta and J. C. Ruiz-Suárez, Phys. Rev. Lett. 92, 114301 (2004). Erratum: ibid 93, 069901(E) (2004).

5. M. Schröter, S. Ulrich, J. Kreft, J.B. Swift, and H.L. Swinney, Phys. Rev. E 74, 011307 (2006).

6. D.V. Kharkar, J.J. McCarthy and J.M. Ottino, CHAOS 9, 594 (1999).

7. P. M. Reis and T. Mullin, Phys. Rev. Lett. 89, 244301 (2002).

8. M. Pica Ciamarra, A. Coniglio, and M. Nicodemi, Phys. Rev. Lett. 94, 188001 (2005).

9. T. Schnautz, R. Brito, C.A. Kruelle, and I. Rehberg, Phys. Rev. Lett. 95, 028001 (2005).

10. H. A. Makse, S. Havlin, P. R. King, and H. E. Stanley, Nature 386, 379 (1997). 
11. K. M. Hill and J. Kakalios, Phys. Rev. E 49, R3610, (1994).

12. D. A. Sanders, M. R. Swift, R. M. Bowley, and P. J. King, Phys. Rev. Lett. 93, 208002 (2004).

13. L. T. Lui, Michael R. Swift, R. M. Bowley, and P. J. King, Phys. Rev. E 75, 051303 (2007).

14. L. Kondic, R.R. Hartley, S.G.K. Tennakoon, B. Painter, and R.P. Behringer, Europhys. Lett. 61, $742(2003)$.

15. D. Serero, I Goldhirsch, S.H. Noskowick and M.-L. Tan, J. Fluid Mech. 554, 237 (2006). The case of different restitution coefficients is treated in Section 5.1.

16. J.J. Brey, M.J. Ruiz-Montero and F. Moreno, Phys. Rev. E 73, 031301 (2006).

17. R.D. Wildman and D.J. Parker, Phys. Rev. Lett. 88, 064301 (2002). R. D. Wildman and J. M. Huntley, Phys Fluids 15, 3090 (2003).

18. K. Feitosa and N. Menon, Phys. Rev. Lett. 88, 198301 (2002).

19. P.E- Krouskop and J. Talbot, Phys. Rev. E 68, 021304 (2003).

20. D. Paolotti, C. Cattuto, U. Marini Bettolo Marconi, and A. Puglisi, Granular Matter 5, 75(2003).

21. V. Garzó, Europhys. Lett. 75, 521 (2006). V. Garzó, Phys. Rev. E, 78, 020301 (2008).

22. R. Brito, H. Enríquez, S. Godoy, and R. Soto, Phys. Rev. E 77, 061301 (2008).

23. R. Soto, Phys. Rev. E 69, 061305 (2004).

24. E. L. Grossman, T. Zhou, and E. Ben-Naim, Phys. Rev. E 55, 4200 (1997).

25. R. Ramírez and R. Soto, Physica A 322, 73 (2003)

26. Ph. A. Martin and J. Piasecki, Europhys. Lett. 46, 613 (1999).

27. A. Barrat, E. Trizac, Granular Matter 4, 57 (2002).

28. B. Meerson, T. Pöschel, and Y. Bromberg, Phys. Rev. Lett. 91, 024301 (2003).

29. N. Burtally, P.J. King, and M.R. Swift, Science 295, 1877 (2002). N. Burtally, P.J. King, M.R. Swift and M. Leaper, Granular Matter 5, 57 (2003). 